smaller sample volumes than other mass spectrometry techniques. The SELDI-TOF-MS-based ProteinChip ${ }^{\circledR}$ technology might be used in future to monitor the immunological reaction between recipient and graft, before the graft becomes damaged. This new technique would aid early treatment with corticosteroids and improve prognosis.

Rachael Williams

Original article Reichelt O et al. (2006) Prediction of renal allograft rejection by urinary protein analysis using ProteinChip arrays (surface-enhanced laser desorption/ionization timeof-flight mass spectrometry). Urology 67: 472-475

\section{The antiviral properties of leflunomide in renal allograft recipients}

Polyoma BK virus nephropathy, which occurs in $3-8 \%$ of renal transplant recipients, can be effectively treated with leflunomide-an immunosuppressant drug commonly used to treat rheumatoid arthritis. This is the exciting conclusion of a small, retrospective analysis of renal allograft recipients with BK nephropathy at the University of Chicago Hospitals and Texas Transplant Institute.

The 26 subjects received either leflunomide plus cidofovir $(n=7)$ or leflunomide alone $(n=19)$. After 6 months, mean BK virus load in the blood and urine was significantly less than at baseline in both groups (both $P<0.001)$. The virus was undetectable in the blood of 11 patients (8 of whom also had no trace of the virus in their urine). During the first 9 months of treatment, mean renal function (determined by serum creatinine levels) did not significantly deteriorate in either group. After 6-40 months of follow-up, graft loss occurred in four patients, all of whom had advanced renal damage or inflammation at baseline. Because the required daily dose of leflunomide was higher than the recommended dose for treating arthritis, blood levels of leflunomide's active metabolite were closely monitored. The dose was reduced if the blood levels of the metabolite consistently exceeded target levels. No serious adverse events were reported.

The mechanisms for the dual immunosuppressant and antiviral activities of this single agent must be identified and might have important implications for the care of renal transplant recipients; currently, the only available strategy for controlling BK viral replication is reducing immunosuppression.

Rachael Williams

Original article Josephson MA et al. (2006) Treatment of renal allograft polyoma BK virus infection with leflunomide. Transplantation 81: 704-710

\section{Reversal of kidney damage in pancreas transplant recipients}

The tubular atrophy and interstitial fibrosis associated with the progression of diabetic and ciclosporin-induced nephropathy have been considered irreversible. New data from eight patients whose type 1 diabetes was cured by pancreatic transplantation have challenged this belief.

Fioretto and colleagues studied tissue from renal biopsies performed 5 and 10 years after pancreas transplantation. After worsening during the first 5 years following transplantation, interstitial fibrosis improved between year 5 and year 10 , primarily as a result of the disappearance of collagen and cellular components. The severity of the initial damage closely correlated with the dose and blood levels of ciclosporin in the first year after transplantation. Reduction in ciclosporin dose over time, prolonged normoglycemia, or both, might have been responsible for the improvement in interstitial fibrosis observed at year 10 .

Tubular atrophy showed a similar trend to interstitial fibrosis. The proportion of atrophic tubules decreased from an abnormal level between 5 and 10 years after transplantation- primarily, the authors propose, because of reabsorption. By contrast, nonatrophic tubules seemed to undergo remodelingas reversal of thickening of the basement membranes-rather than reabsorption.

The authors conclude that although the mechanisms underlying the process remain unclear, the kidney has the capacity to reverse tubular and interstitial damage; arteriolar hyalinosis, however, seems not to be reversible, as no changes in this feature were detected during the study. Understanding the regulation of kidney repair could lead to new therapies for chronic kidney disease.

Original article Fioretto P et al. (2006) Remodeling of Jim Casey renal interstitial and tubular lesions in pancreas transplant recipients. Kidney Int 69: 907-912 